Article

\title{
Clinical, Radiological, and Laboratory Features of Spinal Cord Involvement in Primary Sjögren's Syndrome
}

\author{
Michaela Butryn ${ }^{1}$, Jens Neumann ${ }^{1}$, Leoni Rolfes ${ }^{2}$, Claudius Bartels ${ }^{1}$, Mike P. Wattjes ${ }^{3}$, \\ Nima Mahmoudi ${ }^{3}$, Tabea Seeliger ${ }^{4}$, Franz F. Konen ${ }^{4}$, Thea Thiele ${ }^{5}{ }^{1}$, Torsten Witte ${ }^{5}$, \\ Sven G. Meuth ${ }^{2}$, Thomas Skripuletz ${ }^{4, *,+}$ and Marc Pawlitzki 1,2,*, $^{\mathbb{D}}$ \\ 1 Department of Neurology, Otto-von-Guericke University, 39120 Magdeburg, Germany; \\ michaela.butryn@med.ovgu.de (M.B.); jens.neumann@med.ovgu.de (J.N.); \\ claudius.bartels@med.ovgu.de (C.B.) \\ 2 Department of Neurology with Institute of Translational Neurology, University Hospital Münster, \\ 41849 Münster, Germany; leoni.rolfes@ukmuenster.de (L.R.); sven.meuth@ukmuenster.de (S.G.M.) \\ 3 Department of Diagnostic and Interventional Neuroradiology, Hannover Medical School, 30625 Hannover, \\ Germany; wattjes.mike@mh-hannover.de (M.P.W.); Mahmoudi.Nima@mh-hannover.de (N.M.) \\ 4 Department of Neurology, Hannover Medical School, 30625 Hannover, Germany; \\ seeliger.tabea@mh-hannover.de (T.S.); konen.felix@mh-hannover.de (F.F.K.) \\ 5 Department of Clinical Immunology and Rheumatology, Hannover Medical School, 30625 Hannover, \\ Germany; Thiele.Thea@mh-hannover.de (T.T.); Witte.Torsten@mh-hannover.de (T.W.) \\ * Correspondence: skripuletz.thomas@mh-hannover.de (T.S.); Marc.Pawlitzki@ukmuenster.de (M.P.) \\ + These authors contributed equally to this work.
}

Received: 31 March 2020; Accepted: 12 May 2020; Published: 14 May 2020

\begin{abstract}
Objective: To identify radiological and laboratory hallmarks in patients with primary Sjögren's syndrome (pSS) presenting with spinal cord involvement. Methods: Clinical and laboratory routine parameters were analyzed in a retrospective multicenter case series of four patients who developed myelitis associated with pSS. Serological and cerebrospinal fluid (CSF) measurements of pSS associated anti-SSA(Ro)-antibodies were initiated, and CSF neurofilament light chain (NFL) levels were assessed. NFL values were compared with results from 15 sex-and age-matched healthy controls. Radiological assessment was performed using multi-sequence spinal cord magnetic resonance imaging. Results: Three of the four patients initially developed neurological signs suggestive of myelitis and were subsequently diagnosed with pSS. All patients presented a longitudinal spinal T2-hyperintense lesion in the cervical spinal cord, whereas only two patients showed pleocytosis and oligoclonal bands in the CSF. Median (range) CSF-NFL levels were significantly elevated in patients compared to controls (6672 pg/mL (621-50,000) vs. $585 \mathrm{pg} / \mathrm{mL}(357-729), p=0.009)$. One patient showed sustained, highly increased NFL levels $(50,000 \mathrm{pg} / \mathrm{mL})$ in the initial assessment when radiological signs of axonal injury were still absent. Anti-SSA(Ro)-antibodies were found in the serum of three patients, while two patients additionally presented intrathecal anti-SSA(Ro)-antibody production. Elevated CSF-NFL levels and intrathecal synthesis of anti-SSA(Ro)-antibodies were associated with a relapsing and treatment-resistant disease course. Conclusion: Inflammatory spinal cord lesions associated with pSS are a rare but serious disease leading to severe disability. NFL and anti-SSA(Ro)-antibodies in CSF might serve as prognostic biomarkers and should be routinely assessed in patients with pSS.
\end{abstract}

Keywords: Sjögren's syndrome; Spinal cord; Neurofilament light chain; Antibodies; Cerebrospinal fluid; Myelitis 


\section{Introduction}

Primary Sjögren's syndrome (pSS) is a chronic autoimmune disease characterized by lymphocytic infiltrations in secretory glands and extraglandular neurological manifestations [1,2]. The affection of the peripheral nervous system is more common than central nervous system (CNS) involvement. In particular, inflammatory spinal cord lesions are rarely present and have proven to be difficult to diagnose and treat [3-5].

Anti-SSA(Ro)-antibodies can be found in approximately $50 \%$ of patients suffering from pSS. These antibodies have a high diagnostic value and seem to be associated with extraglandular disease manifestations, although seronegative patients with CNS involvement have been described [6-9]. Interestingly, a direct pathogenic effect has been proposed, as anti-SSA(Ro)-antibodies can be detected serologically in patients with recurrent myelitis and without previous diagnosis of pSS [10].

The levels of neurofilament light chain (NFL) in serum or cerebrospinal fluid (CSF) are thought to reflect neuroaxonal damage and are proposed to have a predictive value concerning outcomes in patients with inflammatory and autoimmune diseases associated with myelitis [11,12].

Thus, we aimed to identify clinical, radiological, and laboratory hallmarks, including new potential biomarkers, in a detailed case series of patients with pSS presenting with spinal cord involvement.

\section{Material and Methods}

\subsection{Patients and Biomaterials}

Patients were recruited at the Departments of Neurology of the University Hospitals Magdeburg, Münster, and Hannover, all located in Germany. CSF and serum samples from 15 sex- and age-matched healthy controls recruited in Magdeburg were included for comparison. All controls reported non-specific complaints and underwent lumbar puncture (LP) during a routine diagnostic examination conducted to rule out any neurological condition. None of the controls was diagnosed with a specific neurological disorder or showed any specific abnormalities during the neurological examination. In addition to the clinical classification, all controls fulfilled the following laboratory criteria defining a normal CSF: $<5$ cells $/ \mu \mathrm{L},<2.0 \mathrm{mmol} / \mathrm{L}$ lactate, no disruption of the blood-CSF-barrier function (defined by the albumin CSF/serum quotient), no oligoclonal bands (OCB), and no intrathecal synthesis of the immunoglobulins (Ig) IgG, IgA, and IgM [13]. In addition, we calculated the antibody specificity indices (ASI) for each case to detect intrathecal anti-SSA(Ro) antibody synthesis. The index is based on the Serum/CSF-quotient of specific antibodies and hereby quantifies their production in the serum vs. in the CSF. It is, therefore, designed to differentiate between passive antibody transmission from serum to CSF (index $\leq 1.5$ ) and intrathecal antibody production (index $>1.5$ ).

\subsection{NFL and Anti-SSA(Ro)-Antibody Analyses}

For all patients, a LP was performed at each respective study site according to standard protocols. The LP for all controls was conducted in Magdeburg. At each study site, CSF cells were counted manually using a Fuchs-Rosenthal chamber. Concentrations of Albumin, IgG, IgM, and IgA in serum and CSF were measured by kinetic nephelometry. Intrathecal synthesis of IgG, IgA, and IgM was calculated according to Reiber's revised hyperbolic function. Isoelectric focusing was applied to detect CSF specific oligoclonal bands. All samples for CSF-NFL and antibody measurements were stored at $-80^{\circ} \mathrm{C}$ and shipped on dry ice. CSF-NFL levels were determined in Magdeburg using commercially available ELISA kits (UmanDiagnostics NF-light@, Umea, Sweden, catalog number 10-7001 CE). Each measurement was performed together with a blank and a commercial positive and negative control provided by the manufacturer. Anti-SSA(Ro)-antibodies both in sera and CSF were measured in Hannover using EliA (ThermoScientific, Waltham, MA USA). Samples were measured in serial procedures and not in batches. 


\subsection{Magnetic Resonance Imaging (MRI) Protocols}

Multi-sequence MRI (1.5 Tesla) of the spinal cord was performed in all patients and included a sagittal T2-weighted turbo-spin echo, a short tau inversion recovery sequence, and a T1-weighted postcontrast sequence covering the cervical and upper thoracic cord. Additionally, axial T2-weighted turbo-spin echo sequences were used. Furthermore, multi-sequence MRI of the head was performed in patients 1, 3, and 4, and included an axial T1- (pre- and postcontrast), a T2-weighted turbo-spin echo sequence and an axial diffusion-weighted imaging sequence. Moreover, a 3D sagittal T2-FLAIR-weighted sequence was applied in patients 1 and 4 and an axial T2-FLAIR-weighted sequence in patient 3 .

\subsection{Standard Protocol Approval, Registration, and Patient Consent}

This study was performed according to the Declaration of Helsinki and approved by the local ethics committees (Magdeburg: No. 07/17, Münster: 2016-053-f-S, Hannover: No. 8270_BO_S_2019). All patients gave written informed consent.

\section{Results}

\subsection{Cohort}

Demographic, clinical, and laboratory findings are provided in Table 1. Four patients from Magdeburg $(n=1)$, Münster $(n=1)$, and Hannover $(n=2)$ with inflammatory spinal cord lesions associated with pSS were enrolled. Three of the four patients were female. The median age at baseline was 51 years (range 39-59 years). Patient 2 was diagnosed with pSS before any neurological symptoms occurred. The remaining three patients developed neurological signs of spinal cord involvement prior to diagnosis. The patients were classified according to the 2017 ACR-EULAR score (American College of Rheumatology/European League against Rheumatic Disease) for primary Sjögren's syndrome and the EULAR Sjögren's syndrome disease activity index (ESSDAI) at baseline [14-16]. Notably, none of the patients fulfilled the current criteria for neuromyelitis optica spectrum disorders. All patients tested negative for aquaporin-4- and myelin oligodendrocyte glycoprotein (MOG)-antibodies [17].

\subsection{Case 1}

A 39-year-old Caucasian female with known hypothyroidism (without evidence of a Hashimoto thyroiditis), alopecia of undeterminable cause, and sicca symptoms developed progressive sensory ataxia of the lower limbs and a mild paraparesis in 10/2017. MRI examinations of the brain and spinal cord were inconspicuous in 10/2017 and 12/2017. Lower limb somatosensory evoked potentials (SEP) showed axonal affection, whereas motor and sensory neurographies provided no evidence of neuropathy. Repeated CSF investigations revealed a pleocytosis $(12-45$ cells $/ \mu \mathrm{L})$ and OCB in CSF (Figure 1A). Immunological analysis of autoantibodies revealed elevated anti-SSA(Ro)-antibodies, while other serum markers for vasculitis or demyelinating CNS disorders were negative. Xerophthalmia and xerostomia were identified, and thus, pSS was diagnosed. Notably, the Schirmer test was not performed at that time (ACR-EULAR score of 3 points at baseline) but turned out positive a few months later. Intravenous (IV) treatment with methylprednisolone ( $1 \mathrm{~g} /$ day for 5 days) in 12/2017 did not lead to clinical improvement. Subsequently, IVIg ( $30 \mathrm{~g} /$ day for 3 days) was administered. Due to a lack of improvement, the patient received six courses of plasmapheresis/immunoadsorption (12/2017-01/2018), leading to temporary clinical stabilization. Three months after disease onset, spinal cord MRI showed a right-sided homogeneous T2-hyperintense lesion without T1-gadolinium enhancement in the posterior column of the cervical spinal cord, which had not been visible on previous MRI scans (Figure 1B,C). The initial MRI of the brain showed a small number of punctate deep white matter lesions suggestive of vascular origin without T1-gadolinium enhancement. 
Table 1. Clinical, radiological, and laboratory findings.

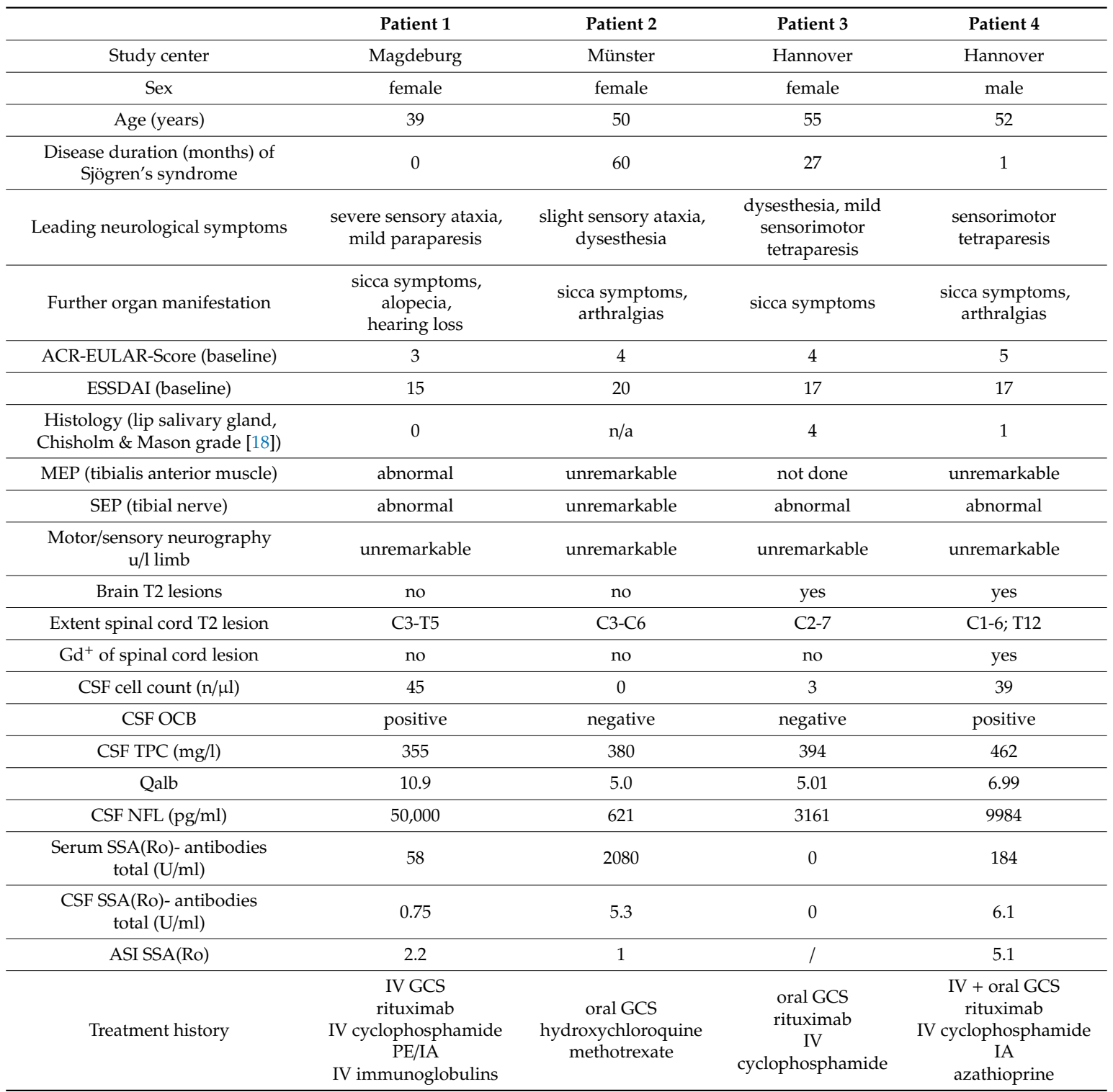

ACR-EULAR-Score = American College of Rheumatology-European League against Rheumatic Disease-Score for primary Sjögren's Syndrome, ASI = antibody specificity index, CSF = cerebrospinal fluid, ESSDAI = EULAR Sjögren Syndrome Disease Activity Index, $\mathrm{Gd}^{+}=$Gadolinium enhancement, GCS = glucocorticosteroids, IA = immunoadsorption, IV = intravenous, NFL = neurofilament light chain, OCB = oligoclonal bands, MEP = Magnetic evoked potentials, n/a: not examined, $\mathrm{PE}=$ Plasma exchange, Qalb = albumin quotient, SEP = Sensory evoked potentials, TPC = total protein count, $\mathrm{u} / 1 \mathrm{limb}=$ upper/lower limb. ASI indicates the CSF/serum difference of antibody amounts per weight unit IgG (normal < 1.5).

During rehabilitation, gait disturbance due to sensory ataxia worsened, leading to wheelchair dependency. Furthermore, the upper limbs were now affected, and the patient developed a bilateral hearing loss. Anti-inflammatory therapy was complemented by two cycles of IV cyclophosphamide (04/2018 and 05/2018, total dose: $900 \mathrm{mg} / \mathrm{m}^{2}$ body surface area). During therapy, an additional left-sided T2-hyperintense lesion, without T1-gadolinium enhancement, was detected in the posterior column of the cervical and thoracic spinal cord (Figure 1C). The right-sided posterior column T2-hyperintense lesion now extended into the thoracic cord. Consequently, two doses of 1000 mg rituximab (05/2018 and 06/2018) were applied. Disease progression could be stopped under ongoing rituximab treatment (every 6 months), but neurological recovery was not achieved. 
A

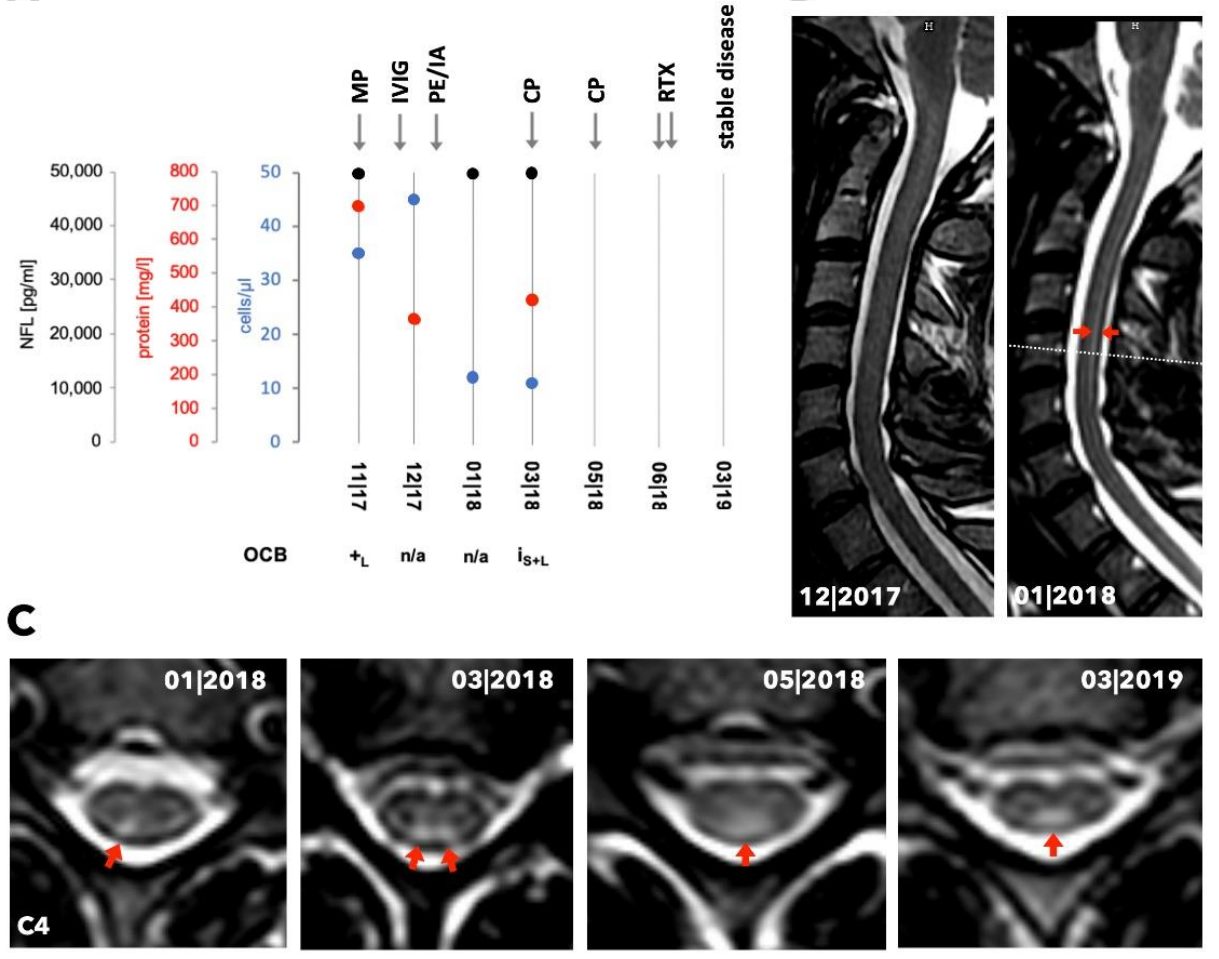

Figure 1. Longitudinal laboratory and radiological data and treatment changes for patient 1. (A) Chart shows the course of cell count, concentrations of protein and neurofilament light chain (NFL) as well as the occurrence of oligoclonal bands (OCB) in the CSF over time (n/a = not examined, ${ }_{\mathrm{L}}=$ detected in CSF, $\mathrm{I}_{\mathrm{S}+\mathrm{L}}=$ identical $\mathrm{OCB}$ in serum and CSF). Arrows indicate time points of treatment $(\mathrm{MP}=$ methylprednisolone, $\mathrm{IVIG}=$ intravenous immunoglobulin, $\mathrm{PE} / \mathrm{IA}=$ plasma exchange/immunoadsorption, $\mathrm{CP}=$ cyclophosphamide, $\mathrm{RTX}=$ rituximab). (B) Panel displays MRI (T2 sagittal plane) at two different time points. The red arrows indicate the T2-enhancement of the posterior part of the spinal cord. The dotted line presents the height of the transversal planes shown in (C). (C) Transversal planes of the cervical spinal cord (C4) show T2-enhancement over time in the posterior columns, indicated by the red arrows.

\subsection{Case 2}

A 50-year-old Caucasian female with pSS, diagnosed in 2014, and evidence of elevated anti-SSA(Ro)-antibodies under treatment with hydroxychloroquine and intermittent use of oral glucocorticosteroids (GCS), developed a mild dysesthesia of the upper and lower limbs and slight sensory ataxia in 2016. Lower limb SEP, peripheral nerve conduction studies of upper and lower limbs, and CSF analysis were inconspicuous. The cervical MRI identified a T2-hyperintense longitudinally extensive spinal cord lesion without T1-gadolinium enhancement in the posterior column as the cause for neurological symptoms (Figure 2C,D). Disease stabilization was achieved with the additional administration of methotrexate on top of the established oral therapy with hydroxychloroquine and GCS.

\subsection{Case 3}

A 55-year-old Caucasian female with known primary biliary cholangitis developed a progressive dysesthesia of the upper and lower limbs and a mild tetraparesis in 2018. The spinal cord MRI showed a long segment T2 lesion without T1-gadolinium-enhancement in the lateral pyramidal tracts and lateral spinothalamic tracts of the cervical spine (Figure 2E,F). The head MRI displayed multiple punctate T2-hyperintense lesions in the deep and periventricular white matter and the dorsal pons. 
Some periventricular and deep white matter lesions showed a perivascular distribution pattern. None of the lesions indicated a disrupted blood-brain barrier.

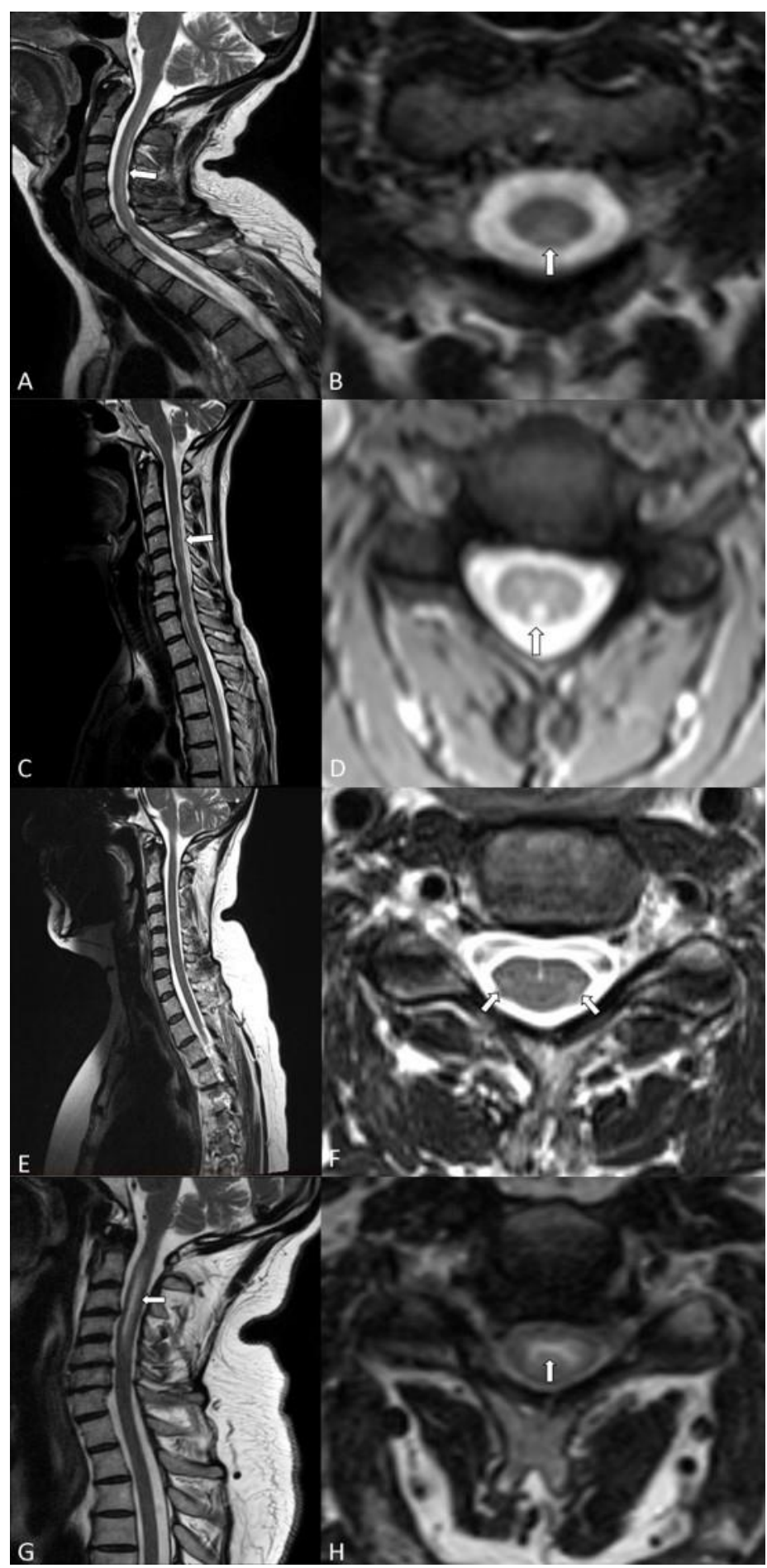

Figure 2. Cervical spinal cord MRI of all patients, with sagittal (left) and axial (right) T2-weighted sequences. (A,B) Patient 1: White arrow indicates a right-sided homogeneous T2-hyperintense lesion in the posterior columns. (C,D) Patient 2: White arrow indicates a T2-hyperintense longitudinally extensive spinal cord lesion in the posterior columns. (E,F) Patient 3: White arrows indicate a long segment T2 lesion of the lateral pyramidal tracts and lateral spinothalamic tracts. $(\mathbf{G}, \mathbf{H})$. Patient 4 : White arrow indicates a long segment T2-hyperintense lesion mainly involving the central grey matter. 
Pathological changes in lower limb SEPs were recorded, whereas nerve conduction examinations of upper and lower limbs showed no impairment of motor and sensory nerves. CSF measurements did not reveal inflammatory signs. In line with the evidence of elevated anti-alpha-fodrin antibodies, objective xerophthalmia, and a pathological minor salivary gland histology, an inflammatory spinal cord lesion associated with pSS was diagnosed. Therapy was initiated with IV cyclophosphamide (cumulative dose $4750 \mathrm{mg} / \mathrm{m}^{2}$ body surface), oral GCS, and finally with rituximab (initially $2 \times 1000 \mathrm{mg}$, hereafter administered every 6 months), under which disease progression could be stopped.

\subsection{Case 4}

A 52-year-old Caucasian male with known psoriasis developed a tetraparesis in 2018. The spinal cord MRI showed a long segment T2-hyperintense lesion with marginal T1-gadolinium-enhancement, mainly involving the central grey matter of the cervical cord (Figure 2G,H). The head MRI presented a small number of punctate deep white matter T2-lesions without T1-gadolinium-enhancement suggestive of vascular origin and a lacunar infarct in the left periventricular white matter. Due to the evidence of elevated anti-SSA(Ro)-antibodies and objective xerophthalmia and xerostomia, myelitis associated with pSS was diagnosed. Lower limb SEPs were conspicuous, and CSF measurements showed a pleocytosis and OCB. Treatment was initiated in $03 / 2018$ with IV corticosteroids $(5 \times 1 \mathrm{~g})$ and 5 cycles of immunoadsorption, followed by one course of IV rituximab $(2 \times 1000 \mathrm{mg})$. Because of disease progression, the administration of IV corticosteroids $(5 \times 1000 \mathrm{mg})$ was repeated without any clinical benefit. Thus, the treatment was escalated to another 5 courses of immunoadsorption and IV cyclophosphamide in $04 / 2018$, under which the neurological symptoms stabilized. Due to relapse in 05/2018, therapy was again escalated with five courses of immunoadsorption and the second cycle of rituximab (1000 mg), resulting in an improvement of the tetraparesis. To achieve long-term clinical stabilization, IV cyclophosphamide was applied at a cumulative dose of $4500 \mathrm{mg} / \mathrm{m}^{2}$ body surface over the next months, and immunosuppressive treatment was hereafter continued with azathioprine while periodic courses of rituximab were administered additionally every 6 months. Disease progression could be stopped.

\subsection{MRI Pattern}

The radiologically examined morphology of the spinal cord revealed a punched-out affection of anatomically defined longitudinal tracts with a long segment involved in all four patients. Patients 1 and 2 presented T2-hyperintense lesions, mainly affecting the dorsal columns of the spinal cord (Figure 2A-D). Patient 3 showed T2-hyperintense lesions in the lateral pyramidal tracts and lateral spinothalamic tracts of the cervical spine on both sides (Figure 2E,F). While seeing primary white matter affection of the spinal cord in patients 1,2, and 3, we detected an isolated long segment T2-hyperintense lesion mainly located in the central grey matter in patient 4 (Figure 2G,H).

\subsection{Antibody Analyses}

Patients 1, 2, and 4 presented elevated levels of anti-SSA(Ro)-antibodies in sera and CSF. An elevated antibody specificity index (ASI) was detectable in patients 1 (2.2) and 4 (5.1), while patient 2 showed no elevated ASI (1.0). We could not detect anti-SSA(Ro)-antibodies in the serum or CSF of patient 3.

\subsection{NFL Analyses}

Although CSF-NFL levels in patient 2 were normal [12], the median (range) CSF-NFL value of pSS patients was significantly higher compared to controls $(6672 \mathrm{pg} / \mathrm{mL}(621-50,000)$ vs. $585 \mathrm{pg} / \mathrm{mL}$ (357-729), Mann-Whitney $U$ test: $p=0.009)$. We initially detected sustained and highly increased NFL levels in patient 1 (permanently at $50,000 \mathrm{pg} / \mathrm{mL}$ ), when imaging signs of axonal injury were still absent (Figure 1A). 


\section{Discussion}

Due to the aggressive disease course involving the CNS, all patients were extensively assessed, including radiological, serological, and CSF analysis.

Previous reports have described a rare occurrence of spinal cord manifestations associated with pSS $[10,19,20]$. Typically, patients present with partly severe neurological symptoms and underlying early longitudinally extensive transverse myelitis as a correlating radiological feature [21]. However, the pattern of spinal cord affection has not yet been fully identified. Our findings regarding the distinct affection of longitudinal spinal tracts suggest a preferred pattern for myelitis associated with pSS. Further studies are needed to verify this MRI pattern. Nevertheless, the presence of such MRI findings should initiate diagnostic procedures of pSS, which include measurement of the tear and saliva flow, anti-SSA(Ro)-antibodies, and, if these are absent, a salivary gland biopsy. Considering the initially normal spinal cord MRI in patient 1 with subsequent spinal cord inflammation during the further disease course, and looking at the delicate structures involved in all our cases, underestimation and delayed diagnosis of this neurological manifestation in patients with pSS is obvious. Additionally, our case series emphasizes that the extent of the spinal cord lesion does not provide prognostic information about the neurological disability, while this association between the clinical state and the length of the spinal cord lesion has been described for other autoantibody-associated demyelinating CNS disorders [22,23].

To date, the relationship between anti-SSA(Ro)-antibodies and neurological manifestations in pSS is not sufficiently understood, in particular since both antibody-negative courses and isolated antibody-positive associated myelitis have been described $[7,8,10,19,24,25]$. Only a few study groups have investigated the impact of CSF-anti-SSA(Ro)-antibodies in patients with neurological involvement of primary rheumatological disorders such as pSS or systemic lupus erythematosus, reporting an intrathecal synthesis occurring partly prior to systemic manifestation $[9,26]$. Due to the severe clinical decline in our two patients with intrathecal synthesis of anti-SSA(Ro)-antibodies, we propose that CSF antibody measurement should be a standard assessment in patients with a suspected neurological manifestation of pSS.

CSF-NFL measurements have recently been discovered to possess biomarker qualities for different neurological disorders and can be applied to assess early axonal damage and reflect early disease activity [27], even in the absence of morphological findings [28]. Interestingly, in our case series, patients with highly elevated CSF-NFL levels showed the most severe clinical course, with multiple relapses and persistent neurological deficits despite aggressive immunosuppressive treatments, comparable to reports of other demyelinating CNS diseases [29-32]. Moreover, for patient 1, the latency between highly increased CSF-NFL levels and the first indicative MRI changes was remarkable [28]. Additionally, the intrathecal synthesis of anti-SSA(Ro)-antibodies was associated with high NFL levels, suggesting antibody-mediated axonal damage [10].

\section{Conclusions}

In conclusion, physicians need to keep in mind that in rare cases, imaging findings can be absent, particularly in the early disease course, or difficult to detect since fine longitudinal tracts can be involved in an isolated manner. However, absent radiological findings should not prevent from further diagnostic procedures necessary to identify a possible pSS. Moreover, our case series implies that CSF-NFL and intrathecal synthesis of anti-SSA(Ro)-antibodies are reliable and early markers for severe spinal cord involvement associated with pSS. We therefore strongly suggest that both values are routinely assessed to predict pSS-related CNS involvement even in the absence of radiological findings or systemic disease activity. Nevertheless, this study is limited because of its low number of analyzed patients, which is a common problem in rare diseases. Further investigations in larger cohorts are needed to verify the pattern of spinal cord affection and to clarify the association between the clinical course, NFL levels and the intrathecal production of anti-SSA(Ro)-antibodies. 
Author Contributions: Conceptualization, T.S. (Thomas Skripuletzand) M.P.; Data curation, M.B., L.R. and C.B.; Formal analysis, M.P.W., T.S. (Thomas Skripuletzand) and M.P.; Investigation, M.B. and M.P.; Methodology, J.N.; Project administration, T.S. (Thomas Skripuletzand); Supervision, T.S. (Thomas Skripuletzand) and M.P.; Validation, J.N., T.S. (Tabea Seeliger), F.F.K., T.T., T.W. and S.G.M.; Visualization, J.N., L.R., M.P.W. and Nima Mahmoudi; Writing—original draft, M.B., T.S. (Thomas Skripuletzand) and M.P.; Writing-review \& editing, J.N., L.R., C.B., M.P.W., N.M., T.S.(Tabea Seeliger), F.F.K., T.T., T.W., S.G.M., T.S. (Thomas Skripuletzand) and M.P. All authors have read and agreed to the published version of the manuscript.

Conflicts of Interest: M.B. received travel/accommodation/meeting expenses from Shire. J.N., C.B., Ta.S., N.M., T.T., F.F.K. report no conflicts of interest. L.R. received travel reimbursements from Merck Serono and Sanofi Genzyme. M.W. received speaker or consultancy honoraria from Bayer Healthcare, Biogen, Biologix, Celgene, Genilac, Imcyse, IXICO, Medison, Merck-Serono, Novartis, Roche, Sanofi-Genzyme. T.W. receives honoraria for lecturing and travel expenses for attending meetings from Aesku.Diagnostics AbbVie, AMGEN, Biogen, BMS, Celgene, Chugai, CSL Behring, Gilead, GSK, Janssen, Lilly, Medac, MSD, Novartis, Octapharma, Pfizer, Roche Pharma, UCB, Sanofi-Aventis. He holds a patent on the diagnostic application of alpha-fodrin antibodies. His research is funded by the Deutsche Forschungsgemeinschaft (DFG, German Research Foundation) under Germany's Excellence Strategy_EXC 2155 “RESIST"-Project ID 39087428. S.G.M. receives honoraria for lecturing and travel expenses for attending meetings from Almirall, Amicus Therapeutics Germany, Bayer Health Care, Biogen, Celgene, Diamed, Genzyme, MedDay Pharmaceuticals, Merck Serono, Novartis, Novo Nordisk, ONO Pharma, Roche, Sanofi-Aventis, Chugai Pharma, QuintilesIMS, and Teva. His research is funded by the German Ministry for Education and Research (BMBF), Deutsche Forschungsgemeinschaft (DFG), Else Kröner Fresenius Foundation, German Academic Exchange Service, Hertie Foundation, Interdisciplinary Center for Clinical Studies (IZKF) Muenster, German Foundation Neurology, and by Almirall, Amicus Therapeutics Germany, Biogen, Diamed, Fresenius Medical Care, Genzyme, Merck Serono, Novartis, ONO Pharma, Roche, and Teva. ThS received honoraria for lecturing from Alexion, Bayer Vital, Biogen, Celgene, CSL Behring, Merck, Novartis, Roche, Sanofi Aventis, all outside the submitted work. M.P. received speaker honoraria and travel/accommodation/meeting expenses from Novartis.

\section{References}

1. Flores-Chávez, A.; Kostov, B.; Solans, R.; Fraile, G.; Maure, B.; Feijoo-Massó, C.; Rascón, F.-J.; Pérez-Alvarez, R.; Zamora-Pasadas, M.; García-Pérez, A.; et al. Severe, life-threatening phenotype of primary Sjögren's syndrome: Clinical characterisation and outcomes in 1580 patients (GEAS-SS Registry). Clin. Exp. Rheumatol. 2018, 36 (Suppl. 112), 121-129.

2. Seeliger, T.; Prenzler, N.K.; Gingele, S.; Seeliger, B.; Körner, S.; Thiele, T.; Bönig, L.; Sühs, K.-W.; Witte, T.; Stangel, M.; et al. Neuro-Sjögren: Peripheral Neuropathy with Limb Weakness in Sjögren's Syndrome. Front. Immunol. 2019, 10, 1600. [CrossRef] [PubMed]

3. Carvajal Alegria, G.; Guellec, D.; Mariette, X.; Gottenberg, J.-E.; Dernis, E.; Dubost, J.-J.; Trouvin, A.-P.; Hachulla, E.; Larroche, C.; Le Guern, V.; et al. Epidemiology of neurological manifestations in Sjögren's syndrome: Data from the French ASSESS Cohort. RMD Open 2016, 2, e000179. [CrossRef] [PubMed]

4. Moreira, I.; Teixeira, F.; Martins Silva, A.; Vasconcelos, C.; Farinha, F.; Santos, E. Frequent involvement of central nervous system in primary Sjögren syndrome. Rheumatol. Int. 2015, 35, 289-294. [CrossRef]

5. Ramos-Casals, M.; Solans, R.; Rosas, J.; Camps, M.T.; Gil, A.; Del Pino-Montes, J.; Calvo-Alen, J.; Jiménez-Alonso, J.; Micó, M.-L.; Beltrán, J.; et al. Primary Sjögren syndrome in Spain: Clinical and immunologic expression in 1010 patients. Medicine (Baltimore) 2008, 87, 210-219. [CrossRef]

6. Fayyaz, A.; Kurien, B.T.; Scofield, R.H. Autoantibodies in Sjögren's Syndrome. Rheum. Dis. Clin. N. Am. 2016, 42, 419-434. [CrossRef]

7. Soliotis, F.; Mavragani, C.; Moutsopoulos, H. Central nervous system involvement in Sjögren's syndrome. Ann. Rheum. Dis. 2004, 63, 616-620. [CrossRef]

8. Tobón, G.J.; Pers, J.-O.; Devauchelle-Pensec, V.; Youinou, P. Neurological Disorders in Primary Sjögren's Syndrome. Autoimmune Dis. 2012, 2012, 645967. [CrossRef]

9. Mégevand, P.; Chizzolini, C.; Chofflon, M.; Roux-Lombard, P.; Lalive, P.H.; Picard, F. Cerebrospinal fluid anti-SSA autoantibodies in primary Sjogren's syndrome with central nervous system involvement. Eur. Neurol. 2007, 57, 166-171. [CrossRef]

10. Hummers, L.K.; Krishnan, C.; Casciola-Rosen, L.; Rosen, A.; Morris, S.; Mahoney, J.A.; Kerr, D.A.; Wigley, F.M. Recurrent transverse myelitis associates with anti-Ro (SSA) autoantibodies. Neurology 2004, 62, 147-149. [CrossRef] 
11. Mariotto, S.; Farinazzo, A.; Monaco, S.; Gajofatto, A.; Zanusso, G.; Schanda, K.; Capra, R.; Mancinelli, C.; Bonora, A.; Bombardi, R.; et al. Serum Neurofilament Light Chain in NMOSD and Related Disorders: Comparison According to Aquaporin-4 and Myelin Oligodendrocyte Glycoprotein Antibodies Status. Mult. Scler. J. Exp. Transl. Clin. 2017, 3, 2055217317743098. [CrossRef]

12. Bridel, C.; van Wieringen, W.N.; Zetterberg, H.; Tijms, B.M.; Teunissen, C.E.; Alvarez-Cermeño, J.C.; Andreasson, U.; Axelsson, M.; Bäckström, D.C.; Bartos, A.; et al. Diagnostic Value of Cerebrospinal Fluid Neurofilament Light Protein in Neurology: A Systematic Review and Meta-analysis. JAMA Neurol. 2019, 76, 1035-1048. [CrossRef] [PubMed]

13. Reiber, H.; Lange, P. Quantification of virus-specific antibodies in cerebrospinal fluid and serum: Sensitive and specific detection of antibody synthesis in brain. Clin. Chem. 1991, 37, 1153-1160. [CrossRef] [PubMed]

14. Shiboski, C.H.; Shiboski, S.C.; Seror, R.; Criswell, L.A.; Labetoulle, M.; Lietman, T.M.; Rasmussen, A.; Scofield, H.; Vitali, C.; Bowman, S.J.; et al. 2016 American College of Rheumatology/European League Against Rheumatism Classification Criteria for Primary Sjögren's Syndrome: A Consensus and Data-Driven Methodology Involving Three International Patient Cohorts. Arthritis Rheumatol. 2017, 69, 35-45. [CrossRef]

15. Seror, R.; Bowman, S.J.; Brito-Zeron, P.; Theander, E.; Bootsma, H.; Tzioufas, A.; Gottenberg, J.-E.; Ramos-Casals, M.; Dörner, T.; Ravaud, P.; et al. EULAR Sjögren's syndrome disease activity index (ESSDAI): A user guide. RMD Open 2015, 1, e000022. [CrossRef]

16. Seror, R.; Ravaud, P.; Bowman, S.J.; Baron, G.; Tzioufas, A.; Theander, E.; Gottenberg, J.-E.; Bootsma, H.; Mariette, X.; Vitali, C. EULAR Sjogren's syndrome disease activity index: Development of a consensus systemic disease activity index for primary Sjogren's syndrome. Ann. Rheum. Dis. 2010, 69, 1103-1109. [CrossRef]

17. Wingerchuk, D.M.; Banwell, B.; Bennett, J.L.; Cabre, P.; Carroll, W.; Chitnis, T.; de Seze, J.; Fujihara, K.; Greenberg, B.; Jacob, A.; et al. International consensus diagnostic criteria for neuromyelitis optica spectrum disorders. Neurology 2015, 85, 177-189. [CrossRef]

18. Chisholm, D.M.; Mason, D.K. Labial salivary gland biopsy in Sjögren's disease. J. Clin. Pathol. 1968, 21, 656-660. [CrossRef]

19. Melikyan, G.; Abdelrahman, M.H.; D’Suoza, A.; Akhtar, N.; Elzouki, A.N.; Hammoudeh, M. Transverse Myelitis Associated with Anti-Ro (SSA) Autoantibodies: A Record of Two Cases. Case Rep. Rheumatol. 2012, 2012, 515768. [CrossRef]

20. Sá, M.J. Acute transverse myelitis: A practical reappraisal. Autoimmun. Rev. 2009, 9, 128-131. [CrossRef]

21. Kahlenberg, J.M. Neuromyelitis optica spectrum disorder as an initial presentation of primary Sjögren's syndrome. Semin. Arthritis Rheum. 2011, 40, 343-348. [CrossRef]

22. Li, R.; Qiu, W.; Lu, Z.; Dai, Y.; Wu, A.; Long, Y.; Wang, Y.; Bao, J.; Hu, X. Acute transverse myelitis in demyelinating diseases among the Chinese. J. Neurol. 2011, 258, 2206-2213. [CrossRef] [PubMed]

23. Jarius, S.; Ruprecht, K.; Kleiter, I.; Borisow, N.; Asgari, N.; Pitarokoili, K.; Pache, F.; Stich, O.; Beume, L.-A.; Hümmert, M.W.; et al. MOG-IgG in NMO and related disorders: A multicenter study of 50 patients. Part 2: Epidemiology, clinical presentation, radiological and laboratory features, treatment responses, and long-term outcome. J. Neuroinflamm. 2016, 13, 280. [CrossRef] [PubMed]

24. Pourmand, N.; Wahren-Herlenius, M.; Gunnarsson, I.; Svenungsson, E.; Löfström, B.; Ioannou, Y.; Isenberg, D.A.; Magnusson, C.G. Ro/SSA and La/SSB specific IgA autoantibodies in serum of patients with Sjögren's syndrome and systemic lupus erythematosus. Ann. Rheum. Dis. 1999, 58, 623-629. [CrossRef] [PubMed]

25. Novak, G.V.; Marques, M.; Balbi, V.; Gormezano, N.W.S.; Kozu, K.; Sakamoto, A.P.; Pereira, R.M.R.; Terreri, M.T.; Magalhães, C.S.; Guariento, A.; et al. Anti-RO/SSA and anti-La/SSB antibodies: Association with mild lupus manifestations in 645 childhood-onset systemic lupus erythematosus. Autoimmun. Rev. 2017, 16, 132-135. [CrossRef]

26. Mevorach, D.; Raz, E.; Steiner, I. Evidence for intrathecal synthesis of autoantibodies in systemic lupus erythematosus with neurological involvement. Lupus 1994, 3, 117-121. [CrossRef]

27. Khalil, M.; Teunissen, C.E.; Otto, M.; Piehl, F.; Sormani, M.P.; Gattringer, T.; Barro, C.; Kappos, L.; Comabella, M.; Fazekas, F.; et al. Neurofilaments as biomarkers in neurological disorders. Nat. Rev. Neurol. 2018, 14, 577-589. [CrossRef] 
28. Pawlitzki, M.; Butryn, M.; Kirchner, F.; Färber, J.; Beuing, O.; Minnerup, J.; Meuth, S.G.; Neumann, J. CSF Neurofilament light chain level predicts axonal damage in cerebral vasculitis. Ann. Clin. Transl. Neurol. 2019, 6, 1134-1137. [CrossRef]

29. Barro, C.; Benkert, P.; Disanto, G.; Tsagkas, C.; Amann, M.; Naegelin, Y.; Leppert, D.; Gobbi, C.; Granziera, C.; Yaldizli, Ö.; et al. Serum neurofilament as a predictor of disease worsening and brain and spinal cord atrophy in multiple sclerosis. Brain 2018, 141, 2382-2391. [CrossRef]

30. Gil-Perotin, S.; Castillo-Villalba, J.; Cubas-Nuñez, L.; Gasque, R.; Hervas, D.; Gomez-Mateu, J.; Alcala, C.; Perez-Miralles, F.; Gascon, F.; Dominguez, J.A.; et al. Combined Cerebrospinal Fluid Neurofilament Light Chain Protein and Chitinase-3 Like-1 Levels in Defining Disease Course and Prognosis in Multiple Sclerosis. Front. Neurol. 2019, 10, 1008. [CrossRef]

31. Kuhle, J.; Plavina, T.; Barro, C.; Disanto, G.; Sangurdekar, D.; Singh, C.M.; Moor, C.; de Engle, B.; Kieseier, B.C.; Fisher, E.; et al. Neurofilament light levels are associated with long-term outcomes in multiple sclerosis. Mult. Scler. 2019, 1352458519885613. [CrossRef] [PubMed]

32. Watanabe, M.; Nakamura, Y.; Michalak, Z.; Isobe, N.; Barro, C.; Leppert, D.; Matsushita, T.; Hayashi, F.; Yamasaki, R.; Kuhle, J.; et al. Serum GFAP and neurofilament light as biomarkers of disease activity and disability in NMOSD. Neurology 2019, 93, e1299-e1311. [CrossRef] [PubMed]

(C) 2020 by the authors. Licensee MDPI, Basel, Switzerland. This article is an open access article distributed under the terms and conditions of the Creative Commons Attribution (CC BY) license (http://creativecommons.org/licenses/by/4.0/). 